\title{
Surface Roughness of Water Measured by X-Ray Reflectivity
}

\section{Citation}

Braslau, A., M. Deutsch, Peter S. Pershan, A. H. Weiss, J. Als-Nielsen, and J. Bohr. 1985. Surface roughness of water measured by x-ray reflectivity. Physical Review Letters 54(2): 114-117.

\section{Published Version}

doi:10.1103/PhysRevLett.54.114

\section{Permanent link}

http://nrs.harvard.edu/urn-3:HUL.InstRepos:10358893

\section{Terms of Use}

This article was downloaded from Harvard University's DASH repository, and is made available under the terms and conditions applicable to Other Posted Material, as set forth at http:// nrs.harvard.edu/urn-3:HUL.InstRepos:dash.current.terms-of-use\#LAA

\section{Share Your Story}

The Harvard community has made this article openly available.

Please share how this access benefits you. Submit a story.

Accessibility 


\title{
Surface Roughness of Water Measured by X-Ray Reflectivity
}

\author{
A. Braslau, M. Deutsch, (a) P. S. Pershan, and A. H. Weiss ${ }^{(b)}$ \\ Division of Applied Sciences, Harvard University, Cambridge, Massachusetts 02138
}

and

J. Als-Nielsen and J. Bohr

Risø National Laboratory, DK-4000 Roskilde, Denmark

(Received 15 October 1984)

\begin{abstract}
The roughness of the liquid-vapor interface for pure water was measured by a technique of x-ray reflectivity. With synchrotron radiation $(\lambda \sim 1.5 \AA)$, the angular dependence of the $x$-ray reflectivity was measured from grazing incidence $(\sim 0.0021 \mathrm{rad})$, where the reflectivity was greater than 0.96 , to an incident angle of $\sim 0.05 \mathrm{rad}$, where the reflectivity was $\sim 7 \times 10^{-8}$. A fit to the data by a theory with only one adjustable parameter obtains $3.2 \AA$ for the root-mean-square roughness of the water surface.
\end{abstract}

PACS numbers: $68.10 . \mathrm{Jy}, 61.10 .-\mathrm{i}$

Although the liquid-vapor interface is of fundamental importance for chemistry and biology, ${ }^{1}$ as well for current surface-physics problems, ${ }^{2-7}$ there have been very few experimental techniques ${ }^{8-11}$ capable of obtaining information on liquid surfaces at the molecular level. Recent synchrotron measurements on the x-ray reflectivity from the free surface of liquid crystals ${ }^{12}$ held out the promise of the technique being generally applicable to the study of all liquid surfaces. ${ }^{13,14} \mathrm{We}$ report here the results of an x-ray-reflectivity measurement in which the root-mean-square roughness of the surface of water averaged over $\sim 10^{7} \AA^{2}$ is shown to be $3.24 \pm 0.05 \AA$. This value is very close to what is expected from thermally excited capillary waves ${ }^{15-20}$ on the water surface at room temperature. We believe this to be the first such measurement of surface roughness of any liquid.

To obtain a good water surface, a circular glass flat $\sim 76 \mathrm{~mm}$ in diameter was thoroughly cleaned in a mixture of hot chromic and sulfuric acids. A thin $(\sim 0.3 \mathrm{~mm})$ water layer approximately $60 \mathrm{~mm}$ in diameter was then applied to the clean surface and the sample was placed inside a sealed stainless steel can $(\sim 10$-cm-diam $\times 6-\mathrm{cm}$ high $)$ with Kapton windows to allow access for the $x$ rays. During the $48-\mathrm{h}$ duration of the measurement, the assembly remained at $\sim 25^{\circ} \mathrm{C}$.

The experiment was carried out with use of synchrotron radiation at $\mathrm{HASYLAB}$ in Hamburg, Germany. Details of the spectrometer have been reported previously. ${ }^{12}$ The geometry is shown schematically in Fig. 1. A monochromatic x-ray beam is extracted from the "white" synchrotron beam by Bragg reflection in the horizontal plane (i.e., out of the plane of the figure) with use of perfect $\mathrm{Si}(111)$ or $\mathrm{Ge}(111)$ single crystals. The monochromatic beam is bent downwards an angle $\theta$ by tilting the crystal $X$. For small angles, both $\theta$ and the beam height are defined by the position $\mathrm{H}_{2}$ and vertical size $h_{2}$ of slit $S_{2}$. To insure that the projection of the beam on the surface of the liquid is less than the sample diameter $d, h_{2}$ must be less than $d \tan \theta$. For other than grazing incidence, it is advantageous to increase $h_{2}$ as $\theta$ increases, since the beam intensity scales directly as $h_{2}$. Table I lists the slit dimensions for various ranges of $\theta$. With the beam defined by $h_{2}$, the measured intensity is normalized to the signal recorded in the ionization detector $M_{2}$. For larger angles $M_{2}$ is removed, and $S_{2}$ is opened up such that the beam height is defined by the height $h_{1}$ of slit $S_{1}$. The intensity is then normalized to the ionization detector $M_{1}$. The width of the beam is defined by $w_{1}$ of slit $S_{1}$ in both cases. Elevators $\mathrm{H}_{2}$ and $\mathrm{H}_{3}$ control the vertical position of slits $S_{2}$ and $S_{3} ; H_{S}$ controls the sample height. The detector $D$ is also mounted on $H_{3}$. For measurement of either the direct beam or for intense signals, corresponding to $\geqslant 5 \%$ reflectivity, a plastic sheet scatters about $0.13 \%$ of the beam into a scintillation detector mounted at $90^{\circ}$ to the incident beam. For weaker signals the scintillation detector is placed directly behind $S_{3}$. It is also possible to rotate the detector about a vertical axis through $H_{S}$ to insure that $S_{3}$ is centered on the reflected beam. Vertical centering is insured by scanning $H_{3}$. For small $\theta$ we confirm that the beam emanating from $S_{2}$ strikes the center of

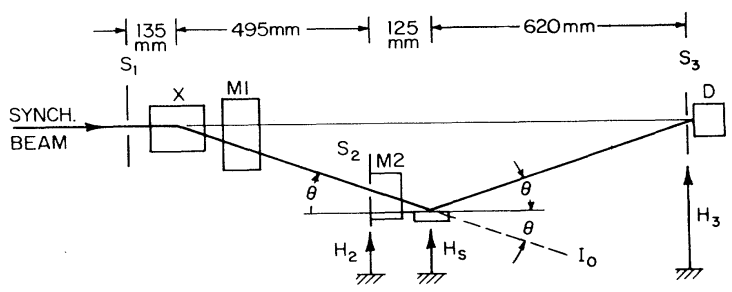

FIG. 1. Schematic representation of the experimental geometry. 
TABLE I. Spectrometer parameters for different ranges of $\theta$. The width of $S 2$ is $14 \mathrm{~mm}$.

\begin{tabular}{cccccc}
\hline \hline \multicolumn{2}{c}{$\theta(\mathrm{rad})$} & $\begin{array}{c}S 1(\mathrm{~mm}) \\
h_{1} \cdot w_{1}\end{array}$ & $\begin{array}{c}S 2(\mathrm{~mm}) \\
h_{2}\end{array}$ & $\begin{array}{c}S 3(\mathrm{~mm}) \\
h_{3} \cdot w_{3}\end{array}$ & Crystal \\
\hline 0.002 & 0.013 & $0.2 \cdot 0.6$ & 0.04 & $0.2 \cdot 1.0$ & $\mathrm{Si}(111)$ \\
0.013 & 0.020 & $0.2 \cdot 0.6$ & 0.1 & $0.2 \cdot 1.0$ & \\
0.020 & 0.030 & $0.6 \cdot 0.6$ & 0.2 & $0.4 \cdot 1.0$ & \\
& 0.030 & $0.6 \cdot 0.6$ & 0.5 & $1.0 \cdot 1.0$ & \\
0.020 & 0.030 & $1.4 \cdot 0.6$ & 0.5 & $1.0 \cdot 1.0$ & \\
0.025 & 0.040 & $0.5 \cdot 0.6$ & 2.0 & $1.0 \cdot 1.0$ & \\
0.025 & 0.050 & $0.5 \cdot 0.6$ & 2.0 & $1.0 \cdot 2.0$ & $\mathrm{Ge}(111)$ \\
\hline \hline
\end{tabular}

the sample by scanning $\mathrm{H}_{3}$ for different positions of $H_{S}$. At the optimum $H_{S}$ one obtains symmetric line shapes with full width at half maximum (FWHM) comparable to that obtained by scanning through the direct beam.

The reflectivity is obtained by comparing the signal at the specular condition to the signal obtained by removing the sample and scanning $\mathrm{H}_{3}$ through $I_{0}$. Figure 2(a) displays such scans for $\theta=0.00244 \mathrm{rad}$. Theoretically a flat surface of water should have essentially $100 \%$ reflectivity for $\cos ^{2} \theta>\cos ^{2} \theta_{c}=1-n e^{2} \lambda^{2} /$ $\pi m c^{2}$ where $n-3.33 \times 10^{23} \mathrm{e} / \mathrm{cm}^{3}$ is the electron density of water. For $\lambda=1.529 \AA, \theta_{c}=0.00264 \mathrm{rad}$. The nearly trapezoidal shape of these two curves agrees very well with the shape expected from the height of $S_{3}(\sim 0.2 \mathrm{~mm}), S_{2}(\sim 0.040 \mathrm{~mm})$, and the vertical size of the synchrotron electron beam $(\sim 1.5 \mathrm{~mm}) 20$ $\mathrm{m}$ from $S_{1}$. If the excess FWHM of $\leqslant 0.0042 \mathrm{~mm}$ is due to surface curvature, it corresponds to a spread of less than $(0.0042 / 620)=6.45 \times 10^{-6} \mathrm{rad}$. The peak reflectivity at this angle of incidence corresponds to $94.7 \%$ reflectivity; the integrated intensity corresponds to $\sim 96.3 \%$ reflectivity.

The reflectivity $R(\theta)$ can be taken to have the form $R(\theta)=R_{F}(\theta)|\Phi(Q)|^{2}$ where $R_{F}(\theta)$ is the optical Fresnel-reflection law for an ideal surface separating materials of dielectric constant $\epsilon=1$ and $\epsilon=\cos ^{2} \theta_{c}<1$, and $\Phi(Q)=n^{-1} \int\langle d n / d z\rangle \exp (i Q z) d z$, with $Q=4 \pi \sin \theta / \lambda^{8}, 12,14,20$ The open squares in Fig. 3 (a) describe the measured reflectivity for $\theta$ varying from $\sim 0.002$ to $\sim 0.050$ rad. Figure $3(b)$ is an expanded version of the same data on a linear scale. The solid line is the theoretical convolution of $R_{F}(\theta)$ with functions that represent the finite, vertical, angular spread of the synchrotron beam, the finite size of the sample, etc. The convolution only differs significantly from $R_{F}(\theta)$ near $\theta_{c}$ where $d R_{F}(\theta) / d \theta$ is large and for $\theta \leqslant 0.8 \theta_{c}$, where the projection of the beam height on the sample surface is comparable to the sample size. Note also that for small $\theta, Q<<1$ and by definition $\Phi(0)=1$; thus $R(\theta)=R_{F}(\theta)$. From the graph

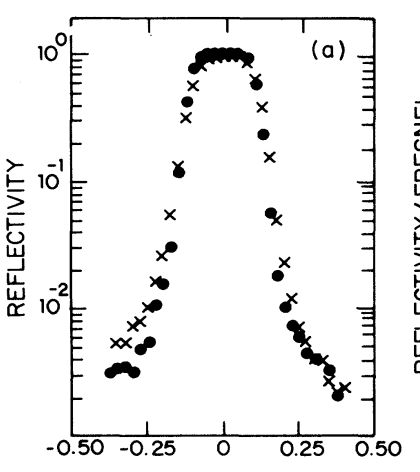

DETECTOR DISPLACEMENT $(\mathrm{mm})$

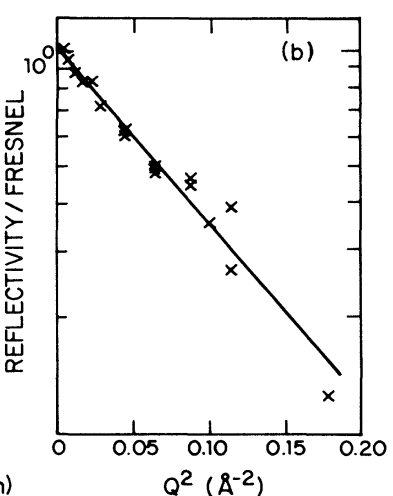

FIG. 2. (a) The reflected intensity vs position $\mathrm{H}_{3}$ of slit $\mathrm{S}_{3}$ for $\theta=0.00244 \mathrm{rad}$ (crosses), and the direct beam $I_{0}$ (solid circles); (b) logarithm of $|\Phi(Q)|^{2}$ vs $Q^{2}$. The solid line is the theoretical fit with $\left\langle u^{2}\right\rangle^{1 / 2}=3.2 \AA$.

shown in Fig. 2(b) of the logarithm of $R(\theta) / R_{F}(\theta)$ vs $Q^{2}$, one can see that $|\Phi(Q)|^{2}$ is well described by the form $|\Phi(Q)|^{2}=\exp \left[-Q^{2}\left\langle u^{2}\right\rangle\right]$ where $\left\langle u^{2}\right\rangle^{1 / 2}=3.24$ $\pm 0.05 \AA$. The dashed line in Fig. 3(a) displays $R_{F}(\theta)|\Phi(Q)|^{2}$.

Strictly speaking $\left\langle u^{2}\right\rangle$ is simply the coefficient of the first nonvanishing term in a power-series expansion of $\ln [\Phi(Q)]$. Alternatively, if the surface roughness is described by Gaussian statistics,

$$
n^{-1}\left\langle\frac{d n}{d z}\right\rangle=\left(2 \pi\left\langle u^{2}\right\rangle\right)^{-1 / 2} \exp \left(\frac{-0.5 z^{2}}{\left\langle u^{2}\right\rangle}\right)
$$

and $\left\langle u^{2}\right\rangle$ is the mean-square roughness. One model takes $\left\langle u^{2}\right\rangle$ to be the mean-square amplitude for capillary waves. ${ }^{15-20}$ If $S_{u u}(\overline{\mathbf{k}})$ is the spectral density for surface displacement $u\left(\overline{\mathbf{r}}_{\perp}\right)$, then $\left\langle u^{2}\right\rangle=\int S_{u u}(\overline{\mathbf{k}}) d^{2} k$ from $|\overline{\mathbf{k}}| \simeq 0$ up to an upper cutoff $\left(k_{\max }\right)$ of the order of $2 \pi$ divided by the molecular diameter. In this model, the reduction in reflectivity as described by $|\Phi(Q)|^{2}$ is caused by scattering by capillary waves. It follows that because of the finite resolution of the spectrometer, small-angle scattering by waves of sufficiently long wavelength will be detected along with the specularly reflected wave. Thus the measured value is

$$
\left\langle u^{2}\right\rangle \simeq \int_{\left(k_{\min }\right)_{x, y}}^{k_{\max }} S_{u u}(\overline{\mathbf{k}}) d^{2} k,
$$

where the long-wavelength limit $\left(k_{\min }\right)_{x, y}^{-1}$ is essentially determined by the resolution of the instrument which, for the present case, is determined by $h_{3}$ and $w_{3}$. The value of $\left\langle u^{2}\right\rangle$ then corresponds to the surface roughness averaged over a coherence area $-4 \pi^{2} /$ $\left(k_{\min }\right)_{x}\left(k_{\min }\right)_{y}$. One can show that for water thickness $h_{s}=0.3 \mathrm{~mm}$, surface tension $\gamma \simeq 73 \mathrm{ergs} / \mathrm{cm}^{2}$, and water density $\rho \simeq 1 \mathrm{~g} / \mathrm{cm}^{2}, \quad|\overline{\mathbf{k}}|>\left(2 \pi / h_{s}\right)$ $\gg(\rho g / \gamma)^{1 / 2} \quad$ and $\quad S_{u u}(\overline{\mathbf{k}}) \simeq\left(k_{\mathrm{B}} T / 4 \pi^{2} \gamma\right)\left(k_{x}^{2}+\right.$ 


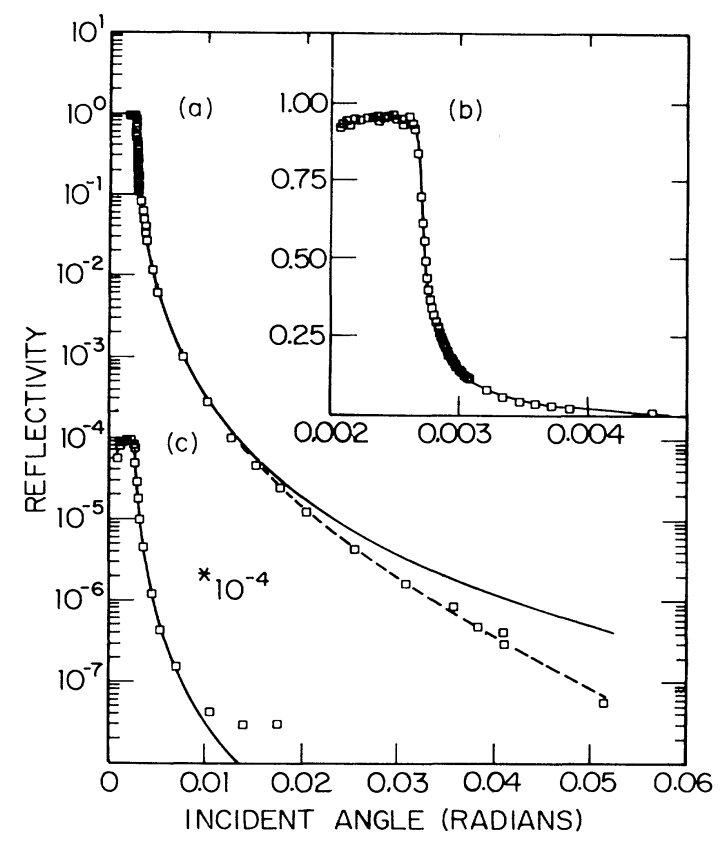

FIG. 3. (a) The measured $x$-ray reflectivity of water (squares) vs $\theta$. The solid line is the convoluted Fresnel form as described in the text. The dashed line includes $|\Phi(Q)|^{2}$. Part (b), expanded version on a linear scale for small $\theta$; part (c), water reflectivity measured with use of a rotating-anode $\mathrm{x}$-ray source. For $\theta \geq 0.01$ the signal is dominated by dark counts.

$\left.k_{y}^{2}\right)^{-1}$. Taking $\left(k_{\min }\right)_{x}=(2 \pi / \lambda) \cdot\left(w_{3} / 1240 \mathrm{~mm}\right)$, $\left(k_{\min }\right)_{y}=(2 \pi / \lambda) \cdot\left(\theta h_{3} / 1240 \mathrm{~mm}\right)$, and $|\overline{\mathbf{k}}|_{\max }=1.63$ $\AA^{-1}$, the calculated $\left\langle u^{2}\right\rangle^{1 / 2}$ varies from $\sim 3.49 \AA$ for the smallest slit at $\theta=0.002 \mathrm{rad}$ to $\sim 2.77 \AA$ for the largest slit at $\theta=0.05 \mathrm{rad}$. On the other hand, since $\exp \left(-Q^{2}\left\langle u^{2}\right\rangle\right) \rightarrow 1$ as $\theta \rightarrow 0$, regardless of the value of $\left\langle u^{2}\right\rangle$, the data for $\theta \leq 0.02 \mathrm{rad}$ or $Q \leq 0.16 \AA^{-1}$ do not have a statistically significant influence on the measured value for $\left\langle u^{2}\right\rangle$. Since the resolution-based variations in predicted values of $\left\langle u^{2}\right\rangle^{1 / 2}$ for $\theta \geq 0.02$ rad only range from 3.06 to $2.77 \AA$, or less than $20 \%$ change in $\left\langle u^{2}\right\rangle$, there is no statistical significance to fitting the data by a value of $\left\langle u^{2}\right\rangle$ that varies with the resolution. The largest slit corresponds to a coherence area of $(1.53)^{2} \cdot(1240)^{2} / 1 \cdot 2 \cdot 0.05 \simeq 3.6 \times 10^{7} \AA^{2}$.

Agreement with the measured value is quite satisfactory and arguments to improve the agreement may be somewhat spurious. Nevertheless, except for introducing $|\overline{\mathbf{k}}|_{\max }$, this calculation neglected the finite dimension of the water molecule. Regardless of any thermal effects, $\langle d n / d z\rangle$ cannot vary rapidly over distances much smaller than the average molecular radius. With the assumption that the contribution to $\left\langle u^{2}\right\rangle$ from molecular size is of the order of the square of the average molecular radius [i.e., $(1.93 \AA)^{2}$ ], an improved estimate would be $(2.77 \AA)^{2}+(1.93 \AA)^{2}$ $=3.37 \AA)^{2}$.

In conclusion, the above agreement between the measured and estimated value of $\left\langle u^{2}\right\rangle$ supports our assertion that we have measured an intrinsic property of the water surface. One implication is that it is possible to study the structural effects of changing the water surface by the addition of surfactants or other chemical species. For example, for liquid-crystal surfaces, even in the isotropic phase, the structure of $|\Phi(Q)|^{2}$ displays features at $Q=2 \pi / 30 \AA^{-1}=0.2 \AA^{-1}$ due to the molecular dimension, ${ }^{12}$ and similar effects should be observable for monolayers of surfactants on water. Other measurements such as studies of critical phenomena on liquid surfaces are equally possible. Although conventional $x$-ray sources do not provide sufficient intensity to obtain the dynamic range reported here, for some purposes synchrotron radiation is not necessary. Figure 3(c) displays data taken with a rotating-anode $x$-ray source and a spectrometer to be described elsewhere. ${ }^{21}$ For $\theta \geq 0.01 \mathrm{rad}$, the signal is dominated by dark counts.

Secondly, the observation of $>96 \%$ reflectivity for $\theta<\theta_{c}$ demonstrates the feasibility of other surface studies based on the existence of an evanescent wave below the water surface. These include structural studies of the two-dimensional solid-liquid transition in surfactant monolayers and use of the phenomena of absorption from the evanescent wave to probe the depth distribution of selective atoms localized near the liquid surface. ${ }^{22-25}$

Finally, improvements that will increase "the measurable" dynamic range of the spectrometer are possible. Presently the angular resolution $\left(\delta \theta^{\prime}\right)$ for the reflected beam is accomplished by a slit rather than a crystal analyzer. The effect is that the background is increased relative to the specularly reflected signal. Since we require that $h_{3}>h_{2}$, the extra intensity obtained by increasing $h_{2}$ is accompanied by a coarsening of the resolution and an increase in background. The addition of a crystal analyzer together with an increase of $-10^{2}$ to $10^{3}$ in synchrotron intensity that is possible with wiggler devices and mirrors will allow measurement of an additional three orders of magnitude in decreased reflectivity. Furthermore, since $R_{F}(\theta)$ is proportional to the square of the electron density, significantly larger values of $Q$ can be measured for liquid metals or other liquids with larger electron densities. An improved spectrometer that will be implemented, with a crystal analyzer to separate angular and spatial resolution, will also allow a systematic study of the dependence of $\left\langle u^{2}\right\rangle$ on $k_{\min }$.

The excellent research conditions provided by HASYLAB, the competent assistance of Risø technical staff E. Dahl Petersen, S. Bang, J. Linderholm, and J. Munck, and discussions with E. Sirota are all grate- 
fully acknowledged.

This work was supported in part by grants from the Danish National Science Foundation, by the Rise $\mathrm{Na}$ tional Laboratory, by the U. S. National Science Foundation through Grants No. DMR 82-12189, No. DMR-80-20247, and No. INT-83-11841, and by the Joint Services Electronics Program (U. S. Army, Navy, and Air Force) through Grant No. N00014-75C-0648.

(a) Permanent address: Physics Department, Bar-Ilan University, Ramat-Gan, Israel.

(b) Permanent address: Physics Department, University of Texas at Arlington, Arlington, Tex. 76019.

${ }^{1}$ See for example, Arthur W. Adamson, Physical Chemistry of Surfaces (Wiley, New York, 1982).

${ }^{2}$ B. Widom, Faraday Symp. Chem. Soc. 1981, 16.

${ }^{3}$ Clive A. Croxton, Statistical Mechanics of the Liquid Surface (Wiley, New York, 1980).

${ }^{4}$ E. Brezin, B. I. Halperin, and S. Leibler, Phys. Rev. Lett. 50, 1387 (1983).

5J. Rudnick and D. Jasnow, Phys. Rev. Lett. 48, 1059 (1982).

${ }^{6}$ R. Lipowsky, Phys. Rev. Lett. 49, 1575 (1982).

${ }^{7}$ M. Robert and C. Stuart, Phys. Rev. Lett. 49, 1434 (1982)

${ }^{8}$ E. S. Wu and W. W. Webb, Phys. Rev. A 8, 2065 (1973).
${ }^{9}$ J. Meunier and D. Langevin, J. Phys. (Paris), Lett. 43, L185 (1982).

${ }^{10}$ D. Beaglehole, Physica (Utrecht) 112B, 320 (1982).

${ }^{11}$ D. S. Sluis, M. P. D'Evelyn, and S. A. Rice, J. Chem. Phys. 78, 1611 (1983).

12P. S. Pershan and J. Als-Nielsen, Phys. Rev. Lett. 52, 759 (1984); J. Als-Nielsen and P. S. Pershan, Nucl. Instrum. Methods 208, 545 (1983); J. Als-Nielsen, F. Christensen, and P. S. Pershan, Phys. Rev. Lett. 48, 1107 (1982).

${ }^{13}$ See Sluis, D'Evelyn, and Rice, Ref. 11, for earlier studies on liquid metals.

${ }^{14}$ David W. Oxtoby, Frank Novak, and Stuart A. Rice, J. Chem. Phys. 76, 5278 (1982).

${ }^{15}$ M. S. Wertheim, J. Chem. Phys. 65, 2377 (1976).

${ }^{16} \mathrm{~J}$. R. Henderson and J. Lekner, Mol. Phys. 36, 781 (1978).

${ }^{17}$ M. H. Kalos, J. K. Percus, and M. Rao, J. Stat. Phys. 17, 111 (1977)

${ }^{18}$ F. B. Buff, R. A. Lovett, and F. H. Stillinger, Phys. Rev. Lett. 15, 621 (1965).

${ }^{19}$ R. A. Lovett, P. W. de Haven, J. J. Vieceli, and F. B. Buff, J. Chem. Phys. 58, 1880 (1973).

20J. Meunier, C. R. Acad. Sci. Ser. II 292, 1469 (1981).

${ }^{21}$ M. Deutsch, A. H. Weiss, P. S. Pershan, and A. Braslau (to be published).

22J. R. Patel and J. A. Golouvchenko, Phys. Rev. Lett. 51, 1469 (1983).

${ }^{23}$ S. Dietrich and H. Wagner, Phys. Rev. Lett. 51, 1469 (1983).

${ }^{24}$ M. Seul, P. Eisenberger, and Harden M. McConnell, Proc. Nat. Acad. Sci. (U.S.A.) 80, 5795 (1983).

${ }^{25}$ I. K. Robinson, Phys. Rev. Lett. 50, 1145 (1983). 